\title{
Enhanced Mean Ratio Estimators of Auxiliary Variables Based on the Linear Mixture of Variances
}

\begin{abstract}
Damisa A. Saddam ${ }^{1}$, Jamila Abdullahi ${ }^{2}$ and Umar Nura ${ }^{3}$
This paper incorporates the variance of auxiliary variables to propose three improved ratio estimators of population mean. To enhance the efficiency of the proposed ratio estimators, a linear combination of the population coefficient of variation, kurtosis, skewness and the population variance of the auxiliary variable is harnessed. The properties relating to the suggested estimators are assessed using constant, bias and mean square error. We also provided practical study for illustration and corroboration using a population data consisting of the fixed capital, which is the supporting variable and output of 80 factories which are the study variables. The suggested improved ratio estimators performed better than other ratio estimators in the literature when compared using bias and mean square error.
\end{abstract}

Keywords: Auxiliary Variable, Kurtosis, Mean Ratio Estimators, Skewness, Variance.

JEL Classification: C02, C13, C83.

DOI: 10.33429/Cjas.09218.1/6

\subsection{Introduction}

Sampling is not just sheer replacement of fractional coverage for a whole coverage.

Sampling is the knowledge, art of governing and determining the consistency of useful statistical information through the concept of probability (Jeelani et al., 2013). The most common method of sampling is the simple random sampling, which is drawn part by part with the same chances of pool for each part at each draw. Ratio estimates encompass the use of well-known population wholes for auxiliary variables to advance the weight of sample values to population approximations of interest. It functions by likening the sample estimate for an auxiliary

\footnotetext{
${ }^{1}$ Corresponding author: Department of Statistics, Ahmadu Bello University, Zaria, Nigeria; asdamisa@abu.edu.ng

${ }^{2}$ Department of Statistics, Ahmadu Bello University, Zaria, Nigeria.

${ }^{3}$ Department of Mathematics and Computer Science, Umaru Musa Yar'adua University, Katshina, Nigeria
} 
variable with known population whole for the same variable on the edge. This is used to modify the sample estimate for the variable of concern. Most times in sample surveys, information on auxiliary variable $X$, along with study variable $Y$ is collected in such a manner that assumes that the two variables are highly associated. This information on auxiliary variable $X$, may well be exploited to acquire a more effective estimator of the population mean. In such cases, the ratio technique of estimation is introduced to explore the information of the auxiliary variable $X$ which is positively associated with the study variable $Y$. This is imperative to reduce bias and increase the precision estimate of the population mean. This can be attained by presenting a large number of modified ratio estimators which exploits the information by well-known values of coefficient of variation, coefficient of skewness, coefficient of kurtosis, median, standard deviation etc. Furthermore, the ratio weights are specified by $X / x$ where $X$ is the recognized population whole for the supporting variable and $x$ is the agreeing estimate of the whole centered on all corresponding units in the sample. Singh (2003), proposed some modified ratio estimators using a linear mixture of coefficient of skewness, coefficient of kurtosis and standard deviation of the auxiliary variable $\mathrm{X}$. To the best of our knowledge, no attempt has been made to develop an estimator that utilizes the linear mixture of identified values of coefficient of variation, kurtosis, skewness and variance of the auxiliary variable. The aim of this study is to propose three improved ratio estimators based on the linear mixture of coefficient of variation, kurtosis, skewness and variance of the auxiliary variable, adapted from existing methodology and to further compare its productivity with existing modified ratio estimators algebraically and empirically using a population data. The population statistics of the data consist of the fixed capital which is denoted by $X$ (supporting variable) and output of 80 factories which are represented by $Y$ (study variable).

Following the introduction is section two which contains the literature review while section three presents methodology of the study. Section four discusses the results while conclusion and policy implications are presented in section five. 


\subsection{Literature Review}

\subsection{Theoretical Framework}

A vast literature on the modification of ratio estimators are available in past studies. Some of these modified ratio estimators are given by Sisodia \& Dwivedi (1981), Singh \& Tailor (2003), Singh \& Tailor (2005), Pandey \& Dubey (1988), Jeelani et al. (2013), Shittu \& Adepoju (2014), Murthy (1967), Cochran (1977), Prasad (1989), Upadhyaya \& Singh (1999), Singh, et al. (2004), Kadilar \& Cingi (2004), Kadilar \& Cingi (2006), Koyuncu \& Kadilar (2009) and Yan \& Tian (2010). These literature can be referred to for further reading. The modified ratio estimators would be compared with the ratio estimators proposed by Abid et al. (2016) which outperformed previous ratio estimators in the literature.

Consider a finite population $Z=Z_{1}, Z_{2}, Z_{3}, \ldots, Z_{n}$ of $N$ different and recognizable bounds. Let $Y$ be the study variable with value $Y_{i}$ of $Z_{i}, i=1,2,3, \ldots, N$ giving a vector $Y=Y_{1}, Y_{2}, \ldots, Y_{N}$. The objective is to evaluate the population mean on the basis of a random sample.

We will mention the estimators in literature with which we are going to make comparisons before discussing about the proposed estimators. Abid et al. (2016) introduced new linear combinations of ratio type estimators in simple random sampling by using non-conventional measures like Hodges Lehman estimator, population mid-range and population tri-mean as supporting information. Estimators proposed by Abid et al. (2016) are given as:

$$
\begin{gathered}
\stackrel{\wedge}{Y}=\frac{\bar{y}+b(\bar{X}-\bar{x})}{(\bar{x}+T M)}(\bar{X}+T M) \\
\stackrel{\wedge}{Y}=\frac{\bar{y}+b(\bar{X}-\bar{x})}{\left(\bar{x} C_{x}+T M\right)}\left(\bar{X} C_{x}+T M\right) \\
\stackrel{\wedge}{Y}=\frac{\bar{y}+b(\bar{X}-\bar{x})}{(\bar{x} \rho+T M)}(\bar{X} \rho+T M) \\
\hat{Y}_{4}=\frac{\bar{y}+b(\bar{X}-\bar{x})}{(\bar{x}+M R)}(\bar{X}+M R)
\end{gathered}
$$




$$
\begin{aligned}
& \stackrel{\wedge}{Y}=\frac{\bar{y}+b(\bar{X}-\bar{x})}{\left(\bar{x} C_{x}+M R\right)}\left(\bar{X} C_{x}+M R\right) \\
& \stackrel{\wedge}{{ }_{6}}=\frac{\bar{y}+b(\bar{X}-\bar{x})}{(\bar{x} \rho+M R)}(\bar{X} \rho+M R) \\
& \frac{\wedge}{Y}=\frac{\bar{y}+b(\bar{X}-\bar{x})}{(\bar{x}+H L)}(\bar{X}+H L) \\
& \underset{8}{\stackrel{\wedge}{Y}}=\frac{\bar{y}+b(\bar{X}-\bar{x})}{\left(\bar{x} C_{x}+H L\right)}\left(\bar{X} C_{x}+H L\right) \\
& \stackrel{\wedge}{\bar{Y}}=\frac{\bar{y}+b(\bar{X}-\bar{x})}{(\bar{x} \rho+H L)}(\bar{X} \rho+H L)
\end{aligned}
$$

The biases, related constants and mean square error (MSE) for Abid et al. (2016) estimators are respectively given by:

$$
\begin{aligned}
& \beta\left(\frac{\hat{\bar{Y}}}{1}\right)=\frac{(1-f) S_{x}^{2}}{n \bar{Y}} R_{1}^{2} ; \quad R_{1}=\frac{\bar{Y}}{\bar{X}+T M} ; \operatorname{MSE}\left(\frac{\wedge}{\bar{Y}}\right) \frac{(1-f)}{n}\left(R_{1}^{2} S_{x}^{2}+S_{y}^{2}\left(1-p^{2}\right)\right) \\
& \beta(\stackrel{\hat{Y}}{1})=\frac{(1-f) S_{x}^{2}}{n \bar{Y}} R_{2}^{2} ; \quad R_{2}=\frac{\bar{Y} C_{x}}{\bar{X} C_{x}+T M} ; \operatorname{MSE}\left(\frac{\wedge}{\bar{Y}}\right) \frac{(1-f)}{n}\left(R_{2}^{2} S_{x}^{2}+S_{y}^{2}\left(1-p^{2}\right)\right) \\
& \beta(\stackrel{\wedge}{\bar{Y}})=\frac{(1-f) S_{x}^{2}}{n \bar{Y}} R_{3}^{2} ; \quad R_{3}=\frac{\bar{Y} p}{\bar{X} p+T M} ; \operatorname{MSE}\left(\frac{\wedge}{\bar{Y}}\right) \frac{(1-f)}{n}\left(R_{3}^{2} S_{x}^{2}+S_{y}^{2}\left(1-p^{2}\right)\right) \\
& \beta\left(\hat{\bar{Y}}_{4}\right)=\frac{(1-f) S_{x}^{2}}{n \bar{Y}} R_{4}^{2} ; R_{1}=\frac{\bar{Y}}{\bar{X}+M R} ; \operatorname{MSE}(\stackrel{\wedge}{\bar{Y}}) \frac{(1-f)}{n}\left(R_{4}^{2} S_{x}^{2}+S_{y}^{2}\left(1-p^{2}\right)\right) \\
& \beta(\stackrel{\wedge}{\bar{Y}})=\frac{(1-f) S_{x}^{2}}{n \bar{Y}} R_{5}^{2} ; R_{5}=\frac{\bar{Y} C_{x}}{\bar{X} C_{x}+M R} ; \operatorname{MSE}\left(\frac{\hat{\bar{Y}}}{5}\right) \frac{(1-f)}{n}\left(R_{5}^{2} S_{x}^{2}+S_{y}^{2}\left(1-p^{2}\right)\right) \\
& \beta\left(\underset{\bar{Y}}{{ }^{\prime}}\right)=\frac{(1-f) S_{x}^{2}}{n \bar{Y}} R_{6}^{2} ; \quad R_{6}=\frac{\bar{Y} p}{\bar{X} p+M R} ; \operatorname{MSE}(\underset{\bar{Y}}{)}) \frac{(1-f)}{n}\left(R_{6}^{2} S_{x}^{2}+S_{y}^{2}\left(1-p^{2}\right)\right) \\
& \beta\left(\frac{\wedge}{\bar{Y}}\right)=\frac{(1-f) S_{x}^{2}}{n \bar{Y}} R_{7}^{2} ; R_{7}=\frac{\bar{Y}}{\bar{X}+H L} ; \operatorname{MSE}\left(\frac{\wedge}{\bar{Y}}\right) \frac{(1-f)}{n}\left(R_{7}^{2} S_{x}^{2}+S_{y}^{2}\left(1-p^{2}\right)\right)
\end{aligned}
$$




$$
\begin{aligned}
& \beta\left(\frac{\hat{\bar{Y}}}{8}\right)=\frac{(1-f) S_{x}^{2}}{n \bar{Y}} R_{8}^{2} ; R_{8}=\frac{\bar{Y} C_{x}}{\bar{X} C_{x}+H L} ; \operatorname{MSE}\left(\frac{\wedge}{\bar{Y}}\right) \frac{(1-f)}{n}\left(R_{8}^{2} S_{x}^{2}+S_{y}^{2}\left(1-p^{2}\right)\right) \\
& \beta\left(\hat{\bar{Y}}_{9}\right)=\frac{(1-f) S_{x}^{2}}{n \bar{Y}} R_{9}^{2} ; R_{9}=\frac{\bar{Y} p}{\bar{X} p+H L} ; \operatorname{MSE}(\stackrel{\wedge}{\bar{Y}}) \frac{(1-f)}{n}\left(R_{9}^{2} S_{x}^{2}+S_{y}^{2}\left(1-p^{2}\right)\right)
\end{aligned}
$$

\subsection{Empirical Literature}

Cochran (1940) proposed a classical ratio type estimator for the estimation of finite population mean using one supporting variable under simple random sampling scheme. Murthy (1967) suggested a product type estimator to calculate the population mean or total of the study variable by using supporting information when coefficient of correlation is negative. The historical development on the improvement of the ratio method of estimation was made by Sen (1993). These improved ratio estimators, although biased, have low mean squared errors as compared with the classical ratio estimator. Upadhyaya and Singh (1999) proposed ratio type estimators with the use of coefficient of variation and coefficient of kurtosis of the auxiliary variable. Kadilar and Cingi (2004) proposed ratio type estimators for the population mean in the simple random sampling with the use of some known auxiliary information on coefficient of kurtosis and coefficient of variation. They showed that their proposed estimators are more efficient than traditional ratio estimator in the estimation of the population mean. Kadilar and Cingi (2006) proposed some modified ratio estimators using known values of coefficient of correlation, kurtosis and coefficient of variation. Yan and Tian (2010) studied the use of coefficient of skewness and coefficient of kurtosis, respectively and showed that it provides better estimates for the population mean in comparison to the usual ratio estimator and different existing estimators. Jeelani et al. (2013) proposed an estimator with the use of coefficient of skewness and quartile deviation of the auxiliary information in the simple random sampling for the estimation of the population mean. Adepoju and Shittu (2013) suggested an improved ratio estimator based on linear mixture of median, coefficients of skewness and kurtosis of the auxiliary variable, which outshone some of the ratio estimators it was compared with using a population data. Shittu and Adepoju (2014) put forward another ratio type estimator using the linear mixture of kurtosis, median and quartile deviation. They evaluated 
its performance using a population data and it performed better than prevailing estimators. Abid et al. (2016) introduced new linear combinations of ratio type estimators in simple random sampling by using non-conventional measures like Hodges Lehman estimator, population mid-range and population tri-mean as supporting information.

\subsection{Methodology}

\subsection{Data}

The data used in this research is a population data of the output of 80 factories denoted by $Y$ with a fixed capital denoted by $X$ in a survey study. Twenty out of the 80 factories were sampled to estimate their productivity. The population statistics of the data is obtainable in page 228 of the book, sampling theory and methods by Murthy (1967). The population statistics of the data were only used for illustrative and empirical purpose in order to evaluate the efficiencies of the proposed estimators of this research. Similarly any other data on trade and investments, market surveys, finance, banking etc., from reliable sources can be used to estimate the population mean of any variable under study as long as there are known values of the auxiliary variable.

Table 1: Descriptive statistics of population data ${ }^{4}$

\begin{tabular}{llll}
\hline \multicolumn{1}{c}{ Population statistics } & & & \\
\hline $\mathrm{N}=80$ & $\mathrm{~S}_{\mathrm{x}}=845.610$ & $\mathrm{D}_{1}=360$ & $\mathrm{D}_{7}=1480$ \\
$\mathrm{n}=20$ & $\mathrm{C}_{\mathrm{x}}=0.751$ & $\mathrm{D}_{2}=460$ & $\mathrm{D}_{8}=1810$ \\
$\bar{Y}=5182.637$ & $\beta_{2}=0.063$ & $\mathrm{D}_{3}=590$ & $\mathrm{D}_{9}=2500$ \\
$\bar{X}=1126.463$ & $\beta_{1}=1.050$ & $\mathrm{D}_{4}=670$ & $\mathrm{D}_{10}=3480$ \\
$\rho=0.941$ & $\mathrm{TM}=931.562$ & $\mathrm{D}_{5}=750$ & $\mathrm{DM}=1052.222$ \\
$\mathrm{~S}_{\mathrm{y}}=1835.659$ & $\mathrm{MR}=1795.5$ & $\mathrm{D}_{6}=850$ & $\mathrm{Md}=757.500$ \\
$\mathrm{C}_{\mathrm{y}}=0.354$ & $S_{x}^{2}=715,056.272$ & $\mathrm{HL}=1040.5$ & $\mathrm{QD}=588.125$ \\
\hline
\end{tabular}

Table 1 shows the descriptive statistics of the population data adopted for the purpose of evaluating the efficiencies of the proposed new estimators and comparing their efficiencies with the existing ones (Murthy, 1967, pp. 228).

\footnotetext{
${ }^{4}$ Source: Murthy (1967, pp. 228)
} 
We propose three new modified ratio estimators by adapting the methodology of Abid et al. (2016), using the linear mixture of coefficient of variation, coefficient of kurtosis, coefficient of skewness and population variance of the supporting variable, on the assumption that the supporting variable is related to the study variable and any linear combination of the information on the supporting variable is utilized to propose new estimators that can be used to estimate the population mean with a reduced MSE. This is shown as follows:

$$
\begin{gathered}
\hat{\bar{Y}}_{p a 1}=\frac{\bar{y}+b(\bar{X}-\bar{x})}{\left.\overline{(x} C_{x}+S_{x}^{2}\right)}\left(\bar{X} C_{x}+S_{x}^{2}\right) \\
\hat{\bar{Y}}_{p a 2}=\frac{\bar{y}+b(\bar{X}-\bar{x})}{\left.\overline{(x} \beta_{2}+S_{x}^{2}\right)}\left(\bar{X} \beta_{2}+S_{x}^{2}\right) \\
\hat{\bar{Y}}_{p a 3}=\frac{\bar{y}+b(\bar{X}-\bar{x})}{\left.\overline{(x} \beta_{1}+S_{x}^{2}\right)}\left(\bar{X} \beta_{1}+S_{x}^{2}\right)
\end{gathered}
$$

The properties of the proposed estimators can be obtained as follows: MSE of these estimators can be gotten using Taylor's series technique defined as

$$
h(\bar{x}, \bar{y}) \cong h(\bar{X}, \bar{Y})+\frac{\partial h(C, d)}{\partial c}\left|\bar{X}, \bar{Y}(\bar{x}-\bar{X})+\frac{\partial h(C, d)}{\partial d}\right| \bar{X}, \bar{Y}(\bar{y}-\bar{Y})
$$

where $h(\bar{x}, \bar{y})=\hat{R}_{p a i}$ and $h(\bar{X}, \bar{Y})=R$.

As shown in Wolter (1985), equation (22) can be applied to the proposed estimators in order to obtain the MSEs as given below:

For the mixture of Coefficient of variation and Variance of the auxiliary variable:

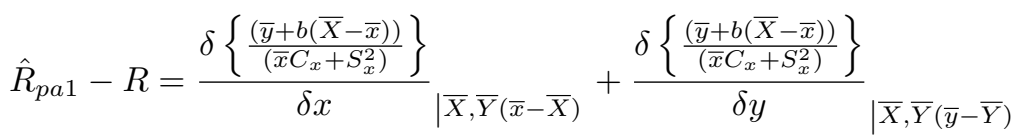

$$
\begin{aligned}
& \hat{R}_{p a 1}-R \cong-\left[\frac{\bar{y}}{\left(\bar{x} C_{x}+S_{x}^{2}\right)^{2}}+\frac{b\left(\bar{X} C_{x}+S_{x}^{2}\right)}{\left(\bar{x} C_{x}+S_{x}^{2}\right)}\right]_{\mid \bar{X}, \bar{Y}(\bar{x}-\bar{X})}+\left.\frac{1}{\left(\bar{x} C_{x}+S_{x}^{2}\right)}\right|_{\mid \bar{X}, \bar{Y}(\bar{y}-\bar{X})} \\
& E\left(\hat{R}_{p a 1}-R\right)^{2} \cong \frac{\left(\bar{Y}+\beta\left(\bar{X} C_{x}+S_{x}^{2}\right)\right)^{2}}{\left(\bar{X} C_{x}+S_{x}^{2}\right)^{2} V(\bar{x})}-\frac{2\left(\bar{Y}+\beta\left(\bar{X} C_{x}+S_{x}^{2}\right)\right)}{\left(\bar{X} C_{x}+S_{x}^{2}\right)^{2} \operatorname{Cov}(\bar{x}, \bar{y})}+\frac{V(\bar{y})}{\left(\bar{x} C_{x}+S_{x}^{2}\right)^{2}}
\end{aligned}
$$




$$
\cong \frac{1}{\left(\bar{x} C_{x}+S_{x}^{2}\right)^{2}}\left\{\frac{\left(\bar{Y}+\beta\left(\bar{X} C_{x}+S_{x}^{2}\right)\right)^{2} V(\bar{x})}{\left(\bar{X} C_{x}+S_{x}^{2}\right)^{2}}-2 \frac{\left(\bar{Y}+\beta\left(\bar{X} C_{x}+S_{x}^{2}\right)\right)}{\left(\bar{X} C_{x}+S_{x}^{2}\right) \operatorname{Cov}(\bar{x}, \bar{y})}+V(\bar{y})\right\}
$$

where $\beta=\frac{S_{x y}}{S_{x}^{2}}=\frac{\rho S_{x} S_{y}}{S_{x}^{2}}=\frac{\rho S_{y}}{S_{x}}$ note we omit the difference of $(E(b)-\beta)$

$$
\begin{gathered}
M S E\left(\bar{y}_{p a 1}\right)=\left(\bar{X} C_{x}+S_{x}^{2}\right)^{2} E(\stackrel{\hat{R}}{p a 1}-R)^{2} \\
\cong \frac{\left(\bar{Y}+\beta\left(\bar{X} C_{x}+S_{x}^{2}\right)\right)^{2}}{\left(\bar{X} C_{x}+S_{x}^{2}\right)^{2}} V(\bar{x})-2 \frac{\left(\bar{Y}+\beta\left(\bar{X} C_{x}+S_{x}^{2}\right)\right)}{\left(\bar{X} C_{x}+S_{x}^{2}\right)} \operatorname{Cov}(\bar{x}, \bar{y})+V(\bar{y}) \\
\cong \frac{\left(\bar{Y}^{2}+2 \beta\left(\bar{X} C_{x}+S_{x}^{2}\right) \bar{Y}+\beta^{2}\left(\bar{X} C_{x}+S_{x}^{2}\right)^{2}\right) V(\bar{x})}{\left(\bar{X} C_{x}+S_{x}^{2}\right)^{2}}-\frac{\left(2 \bar{Y}+2 \beta\left(\bar{X} C_{x}+S_{x}^{2}\right)\right.}{\left.\left(\bar{X} C_{x}+S_{x}^{2}\right)\right) \operatorname{Cov}(\bar{x}, \bar{y})}+V(\bar{y}) \\
\cong \frac{(1-f)}{n}\left\{\left[\frac{\bar{Y}^{2}}{\left(\bar{X} C_{x}+S_{x}^{2}\right)^{2}}+\frac{2 \beta \bar{Y}}{\left(\bar{X} C_{x}+S_{x}^{2}\right)}+\beta^{2}\right] S_{x}^{2}-\left[\frac{2 \bar{Y}}{\left(\bar{X} C_{x}+S_{x}^{2}\right)}+2 \beta\right] S_{x y}\right\} \\
\cong \frac{(1-f)}{n}\left(R_{p a 1}^{2} S_{x}^{2}+2 \beta R_{p a 1} S_{x}^{2}+\beta^{2} S_{x}^{2}-2 R_{p a 1} S_{x y}-2 \beta S_{x y}+S_{y}^{2}\right) \\
M S E\left(\bar{y}_{p a 1}\right) \cong \frac{(1-f)}{n}\left(R_{p a 1}^{2} S_{x}^{2}+2 R_{p a 1} \rho S_{x} S_{y}+\rho^{2} S_{y}^{2}-2 R_{p a 1} S_{x y}-2 R_{p a 1} \rho S_{x} S_{y}\right) \\
-\frac{(1-f)}{n} S_{y}^{2}\left(2 \rho^{2} S_{y}^{2}+S_{y}^{2}\right) \\
\cong \frac{(1-f)}{n}\left(R_{p a 1}^{2} S_{x}^{2}-\rho^{2} S_{y}^{2}+S_{y}^{2}\right) \cong \frac{(1-f)}{n}\left(R_{p a 1}^{2} S_{x}^{2}+S_{y}\left(1-\rho^{2}\right)\right)
\end{gathered}
$$

For the mixture of Coefficient of Kurtosis and Variance of the auxiliary variable:

$$
\begin{gathered}
\hat{R}_{p a 2}-R \cong \frac{\delta[(\bar{y}+b(\bar{X}-\bar{x}))] /[(\bar{y}+b(\bar{X}-\bar{x}))]}{\delta \bar{x}} \mid \bar{X}, \bar{Y}(\bar{x}-\bar{X}) \\
+\frac{\delta[(\bar{y}+b(\bar{X}-\bar{x}))] /\left(\bar{x} \beta_{2}+S_{x}^{2}\right)}{\delta \bar{x}} \mid \bar{X}, \bar{Y}(\bar{y}-\bar{Y}) \\
\hat{R}_{p a 2}-R \cong-\left[\frac{\bar{y}}{\left(\bar{x} \beta_{2}+S_{x}^{2}\right)^{2}}+\frac{b\left(\bar{X} \beta_{2}+S_{x}^{2}\right)}{\left(\bar{x} \beta_{2}+S_{x}^{2}\right)}\right]\left|\bar{X}, \bar{Y}(\bar{x}-\bar{X})+\frac{1}{\left(\bar{x} \beta_{2}+S_{x}^{2}\right)}\right| \bar{X}, \bar{Y}(\bar{y}-\bar{Y}) \\
E\left(\hat{R}_{p a 2}-R\right)^{2} \cong \frac{\left(\bar{Y}+\beta\left(\bar{X} \beta_{2}+S_{x}^{2}\right)\right)^{2}}{\left(\bar{X} \beta_{2}+S_{x}^{2}\right)^{2}} V(\bar{x})-\frac{2\left(\bar{Y}+\beta\left(\bar{X} \beta_{2}+S_{x}^{2}\right)\right)}{\left(\bar{X} \beta_{2}+S_{x}^{2}\right)} \operatorname{Cov}(\bar{x}, \bar{y})
\end{gathered}
$$




$$
\begin{gathered}
+\frac{1}{\left(\bar{X} \beta_{2}+S_{x}^{2}\right)^{2}} V(\bar{y}) \\
\cong \frac{1}{\left(\bar{x} \beta_{2}+S_{x}^{2}\right)^{2}}\left\{\frac{\left(\bar{Y}+\beta\left(\bar{X} \beta_{2}+S_{x}^{2}\right)\right)^{2}}{\left(\bar{X} \beta_{2}+S_{x}^{2}\right)^{2}} V(\bar{x})-\frac{2\left(\bar{Y}+\beta\left(\bar{X} C_{x}+S_{x}^{2}\right)\right)}{\left(\bar{X} \beta_{2}+S_{x}^{2}\right)} \operatorname{Cov}(\bar{x}, \bar{y})+V(\bar{y})\right\}
\end{gathered}
$$

where $\beta=\frac{S_{x y}}{S_{x}^{2}}=\frac{\rho S_{x} S_{y}}{S_{x}^{2}}=\frac{\rho S_{y}}{S_{x}}$ note we omit the difference of $(E(b)-\beta)$

$$
\begin{gathered}
M S E\left(\bar{y}_{p a 2}\right)=\left(\bar{X} \beta_{2}+S_{x}^{2}\right)^{2} E\left(\underset{p a 2}{\stackrel{\wedge}{R}-R)^{2}}\right. \\
\cong \frac{\left(\bar{Y}+\beta\left(\bar{X} \beta_{2}+S_{x}^{2}\right)\right)^{2}}{\left(\bar{X} C_{x}+S_{x}^{2}\right)^{2}} V(\bar{x})-\frac{2\left(\bar{Y}+\beta\left(\bar{X} \beta_{2}+S_{x}\right)\right)}{\left(\bar{X} C_{x}+S_{x}^{2}\right)} \operatorname{Cov}(\bar{x}, \bar{y})+V(\bar{y}) \\
\cong \frac{\bar{Y}^{2}+2 B\left(\bar{X} \beta_{2}+S_{x}^{2}\right) \bar{Y}+\beta^{2}\left(\bar{X} \beta_{2}+S_{x}^{2}\right)^{2}}{\left(\bar{X} \beta_{2}+S_{x}^{2}\right)^{2} V(\bar{x})}-\frac{2 \bar{Y}+2 B\left(\bar{X} \beta_{2}+S_{x}^{2}\right) C o v(\bar{x}, \bar{y})}{\left(\bar{X} \beta_{2}+S_{x}^{2}\right)}+V(\bar{y}) \\
\cong \frac{(1-f)}{n}\left\{\left[\frac{\bar{Y}^{2}}{\left(\bar{X} \beta_{2}+S_{x}^{2}\right)^{2}}+\frac{2 \beta \bar{Y}}{\left(\bar{X} \beta_{2}+S_{x}^{2}\right)}+\beta^{2}\right] S_{x}^{2}-\left[\frac{2 \bar{Y}}{\left(\bar{X} \beta_{2}+S_{x}^{2}\right)}+2 \beta\right] S_{x y}+S_{y}^{2}\right\} \\
\cong \frac{(1-f)}{n}\left(R_{p a 2}^{2} S_{x}^{2}-2 \beta R_{p a 2} S_{x}^{2}+\beta^{2} S_{x}^{2}-2 R_{p a 2} S_{x y}-2 \beta S_{x y}+S_{y}^{2}\right) \\
M S E\left(\bar{y}_{p a 2}\right) \cong \frac{(1-f)}{n}\left(R_{p a 2}^{2} S_{x}^{2}+2 R_{p a 2} \rho S_{x} S_{y}+\rho^{2} S_{y}^{2}-2 R_{p a 2} \rho S_{x} S_{y}-2 \rho^{2} S_{y}^{2}+S_{y}^{2}\right) \\
\cong \frac{(1-f)}{n}\left(R_{p a 2}^{2} S_{x}^{2}-\rho^{2} S_{y}^{2}+S_{y}^{2}\right) \cong \frac{(1-f)}{n}\left(R_{p a 2}^{2} S_{x}^{2}+S_{y}\left(1-\rho^{2}\right)\right)
\end{gathered}
$$

For the mixture of Coefficient of Skewness and Variance of the auxiliary variable:

$$
\begin{gathered}
\hat{R}_{p a 3}-R \cong-\left[\frac{\bar{y}}{\left(\bar{x} \beta_{1}+S_{x}^{2}\right)^{2}}+\frac{b\left(\bar{X} \beta_{1}+S_{x}^{2}\right)}{\left(\bar{x} \beta_{1}+S_{x}^{2}\right)}\right]\left|\bar{X}, \bar{Y}(\bar{x}-\bar{X})+\frac{1}{\left(\bar{x} \beta_{1}+S_{x}^{2}\right)}\right| \bar{X}, \bar{Y}(\bar{y}-\bar{Y}) \\
\hat{R}_{p a 3}-R \cong-\left[\frac{\bar{y}}{\left(\bar{x} \beta_{1}+S_{x}^{2}\right)^{2}}+\frac{b\left(\bar{X} \beta_{1}+S_{x}^{2}\right)}{\left(\bar{x} \beta_{1}+S_{x}^{2}\right)}\right]\left|\bar{X}, \bar{Y}(\bar{x}-\bar{X})+\frac{1}{\left(\bar{x} \beta_{1}+S_{x}^{2}\right)}\right| \bar{X}, \bar{Y}(\bar{y}-\bar{Y}) \\
E\left(\hat{R}_{p a 3}-R\right)^{2} \cong \frac{\left(\bar{Y}+\beta\left(\bar{X} \beta_{1}+S_{x}^{2}\right)\right)^{2} V(\bar{x})}{\left(\bar{X} \beta_{1}+S_{x}^{2}\right)^{4}}-\frac{2\left(\bar{Y}+\beta\left(\bar{X} \beta_{1}+S_{x}^{2}\right)\right) \operatorname{Cov}(\bar{x}, \bar{y})}{\left(\bar{X} \beta_{1}+S_{x}^{2}\right)^{3}} \\
+\frac{V(\bar{y})}{\left(\bar{X} \beta_{1}+S_{x}^{2}\right)^{2}}
\end{gathered}
$$




$$
\cong \frac{1}{\left(\bar{x} C_{x}+S_{x}^{2}\right)^{2}}\left\{\frac{\left(\bar{Y}+\beta\left(\bar{X} \beta_{1}+S_{x}^{2}\right)\right)^{2} V(\bar{x})}{\left(\bar{X} \beta_{1}+S_{x}^{2}\right)^{2}}-\frac{2\left(\bar{Y}+\beta\left(\bar{X} \beta_{1}+S_{x}^{2}\right)\right) \operatorname{Cov}(\bar{x}, \bar{y})}{\left(\bar{X} \beta_{1}+S_{x}^{2}\right)}+V(\bar{y})\right\}
$$

where $\beta=\frac{S_{x y}}{S_{x}^{2}}=\frac{\rho S_{x} S_{y}}{S_{x}^{2}}=\frac{\rho S_{y}}{S_{x}}$ note we omit the difference of $(E(b)-\beta)$

$$
\begin{gathered}
M S E\left(\bar{y}_{p a 3}\right)=\left(\bar{X} \beta_{1}+S_{x}^{2}\right)^{2} E(\underset{p a 3}{R}-R)^{2} \\
\cong \frac{\left(\bar{Y}+\beta\left(\bar{X} \beta_{1}+S_{x}^{2}\right)\right)^{2}}{\left(\bar{X} \beta_{1}+S_{x}^{2}\right)^{2}} V(\bar{x})-\frac{2\left(\bar{Y}+\beta\left(\bar{X} \beta_{1}+S_{x}\right)\right)}{\left(\bar{X} \beta_{1}+S_{x}^{2}\right)} \operatorname{Cov}(\bar{x}, \bar{y})+V(\bar{y}) \\
\cong \frac{\bar{Y}^{2}+2 \beta\left(\bar{X} \beta_{1}+S_{x}^{2}\right) \bar{Y}+\beta^{2}\left(\bar{X} \beta_{1}+S_{x}^{2}\right)^{2}}{\left(\bar{X} \beta_{1}+S_{x}^{2}\right)^{2}} V(\bar{x})-\frac{2 \bar{Y}+2 \beta\left(\bar{X} \beta_{1}+S_{x}^{2}\right)}{\left(\bar{X} \beta_{1}+S_{x}^{2}\right)} \operatorname{Cov}(\bar{x}, \bar{y})+V(\bar{y}) \\
\cong \frac{(1-f)}{n}\left\{\left[\frac{\bar{Y}^{2}}{\left(\bar{X} \beta_{1}+S_{x}^{2}\right)^{2}}+\frac{2 \beta \bar{Y}}{\left(\bar{X} \beta_{1}+S_{x}^{2}\right)}+\beta^{2}\right] S_{x}^{2}-\left[\frac{2 \bar{Y}}{\left(\bar{X} \beta_{1}+S_{x}^{2}\right)}+2 \beta\right] S_{x y}+S_{y}^{2}\right\} \\
\cong \frac{(1-f)}{n}\left(R_{p a 3}^{2} S_{x}^{2}-2 \beta R_{p a 3} S_{x}^{2}+\beta^{2} S_{x}^{2}-2 R_{p a 3} S_{x y}-2 \beta S_{x y}+S_{y}^{2}\right) \\
M S E\left(\bar{y}_{p a 3}\right) \cong \frac{(1-f)}{n}\left(R_{p a 3}^{2} S_{x}^{2}+2 R_{p a 3} \rho S_{x} S_{y}+\rho^{2} S_{y}^{2}-2 R_{p a 3} \rho S_{x} S_{y}-2 \rho^{2} S_{y}^{2}+S_{y}^{2}\right) \\
\cong \frac{(1-f)}{n}\left(R_{p a 3}^{2} S_{x}^{2}-\rho^{2} S_{y}^{2}+S_{y}^{2}\right) \cong \frac{(1-f)}{n}\left(R_{p a 3}^{2} S_{x}^{2}+S_{y}\left(1-\rho^{2}\right)\right)
\end{gathered}
$$

Similarly, the constant, bias and MSE are obtained as;

$$
\begin{gathered}
\operatorname{Bias}\left(\bar{y}_{p a 1}\right) \cong \frac{(1-f)}{n} \frac{S_{x}^{2}}{\bar{Y}} R_{p a 1}^{2} \\
\beta\left(\bar{Y}_{p a i}^{\wedge}\right)=\frac{(1-f)}{n} \frac{S_{x}^{2}}{\bar{Y}} R_{p a i}^{2} \quad i=1,2,3 \\
R_{p a 1}=\frac{\bar{Y} C_{x}}{\left(\bar{X} \beta_{1}+S_{x}^{2}\right)} \\
R_{p a 2}=\frac{\bar{Y} \beta_{2}}{\left(\bar{X} \beta_{1}+S_{x}^{2}\right)} \\
R_{p a 3}=\frac{\bar{Y} \beta_{1}}{\left(\bar{X} \beta_{2}+S_{x}^{2}\right)}
\end{gathered}
$$




$$
\begin{aligned}
M S E(\stackrel{\hat{Y}}{\text { pai }}) & =\frac{(1-f)}{n}\left(R_{\text {pai }}^{2} S_{x}^{2}+S_{y}^{2}\left(1-\rho^{2}\right)\right) \quad i=1,2,3 \\
P R E & =\frac{M S E(\text { existing }- \text { estimator })}{M S E(\text { proposed }- \text { estimator })} * 100
\end{aligned}
$$

\subsection{Productivity Evaluations of the Proposed Estimators}

In this segment, the circumstance under which the suggested improved ratio estimators will have least mean square error compared with the classical ratio estimator and prevailing adjusted ratio estimators to evaluate the finite population mean would be shown algebraically. Contrast with Conventional Mean Ratio Estimator from the expression of suggested and conventional ratio estimators MSEs, we would show the circumstances for which the suggested estimators are more efficient than the ratio estimators:

$$
\begin{aligned}
M S E\left(\stackrel{\bar{Y}}{p a i}^{\wedge}\right) & \leq \operatorname{MSE}(\underset{r}{\wedge}) \\
\frac{(1-f)}{n}\left(R_{p a i}^{2} S_{x}^{2}+S_{y}^{2}\left(1-\rho^{2}\right)\right) & \leq \frac{(1-f)}{n}\left(R_{i}^{2} S_{x}^{2}+S_{y}^{2}\left(1-\rho^{2}\right)\right) \\
R_{p a i}^{2} S_{x}^{2} & \leq R_{i}^{2} S_{x}^{2} \\
R_{p a i} & \leq R_{i}
\end{aligned}
$$

\subsection{Practical Study}

The performances of the suggested ratio estimators are assessed and compared with the prevailing ratio estimators by using a population data given in Murthy (1967), the fixed capital is denoted by X (supporting variable) and output of 80 factories are represented by Y (study variable). The population statistics of the data is available in Table 1.

\subsection{Discussion of Results}

We applied the proposed and existing estimators to the population statistics given in Table 1. Table 2 shows results of the existing estimators labelled $\hat{\bar{Y}}_{1-9}$ and the proposed estimators using Kurtosis $(k t)$, Skewness $(s k)$ and Coefficient of Variation $(c v)$, when applied to the population data given in Murthy (1967). The related constant, Bias and Mean Square Error (MSE) of the three proposed estimators are all smaller when compared to the existing ones. 
Table 2: The statistical analysis of the estimators for the population statistics

\begin{tabular}{llll}
\hline Estimators & Related Constant & Bias & MSE \\
\hline$\hat{\bar{Y}}_{1}$ & 2.518 & 32.81 & $184,446.20$ \\
$\hat{\bar{Y}}_{2}$ & 2.189 & 24.79 & $142,903.20$ \\
$\hat{\bar{Y}}_{3}$ & 2.449 & 31.03 & $175,238.70$ \\
$\hat{\bar{Y}}_{4}$ & 1.774 & 16.23 & $98,755.61$ \\
$\hat{\bar{Y}}_{5}$ & 1.473 & 11.23 & $72,582.52$ \\
$\hat{\bar{Y}}_{6}$ & 1.708 & 15.10 & $92,644.60$ \\
$\hat{\bar{Y}}_{7}$ & 2.392 & 29.52 & $167,778.60$ \\
$\hat{\bar{Y}}_{8}$ & 2.063 & 22.01 & $126,487.60$ \\
$\hat{\bar{Y}}_{9}$ & 2.372 & 27.90 & $158,990.70$ \\
Proposed $_{\text {(kt) }}$ & $\mathbf{0 . 0 0 0 4}$ & $\mathbf{0 . 0 0 0 0 0 1}$ & $\mathbf{1 4 , 4 7 0 . 8 1}$ \\
Proposed(sk) $_{\text {Proposed (cv) }}$ & $\mathbf{0 . 0 0 7 6}$ & $\mathbf{0 . 0 0 0 3 0 0}$ & $\mathbf{1 4 , 4 7 2 . 3 6}$ \\
\hline & $\mathbf{0 . 0 0 5 4}$ & $\mathbf{0 . 0 0 0 1 5 1}$ & $\mathbf{1 4 , 4 7 1 . 5 9}$ \\
\hline
\end{tabular}

Table 3 shows the Percentage Relative Efficiency (PRE) values of the proposed modified estimators to the existing ones when applied to the population data given in Murthy (1967). The results show that the new modified estimators are at least five times more efficient than the existing ones.

Table 3: Percentage Relative Efficiency (PRE) of the proposed estimators with the estimators in Abid et al. (2016).

\begin{tabular}{|c|c|c|c|}
\hline Estimators & Proposed (kt) & proposed (sk) & proposed (cv) \\
\hline$\overline{\bar{Y}_{1}}$ & 1274.61 & 1274.47 & 1274.54 \\
\hline$\widehat{\hat{\bar{Y}}_{2}}$ & 987.53 & 987.42 & 987.47 \\
\hline$\hat{\bar{Y}}_{3}$ & 1210.98 & 1210.85 & 1210.92 \\
\hline $\bar{Y}_{4}$ & 682.45 & 682.37 & 682.41 \\
\hline $\bar{Y}_{5}$ & 501.58 & 501.53 & 501.55 \\
\hline$\overline{\bar{Y}}_{6}$ & 640.22 & 640.15 & 640.18 \\
\hline $\bar{Y}_{7}$ & 1159.43 & 1159.3 & 1159.73 \\
\hline $\bar{Y}_{8}$ & 874.09 & 873.99 & 874.04 \\
\hline
\end{tabular}

The accessibility of auxiliary data improves the productivity of the estimators. The mean ratio estimator has been suggested using well-known values of population 
variance, kurtosis, skewness and coefficient of variation of the auxiliary variable. The performance of the proposed modified mean ratio estimators and the existing estimators has been evaluated using the data from Murthy (1967). The population statistics are shown in Table 1 . The properties of the proposed and existing estimators have been studied through their related constants, bias and mean squared errors. The bias of the suggested estimators are almost unbiased as the estimates from the population data are approximately approaching zero which gives the suggested estimators an edge over the prevailing ones. The mean squared errors of the proposed estimators based on the mixtures of coefficient of variation, kurtosis, skewness and variance of the auxiliary variable performed better than all the existing estimators proposed by Abid et al. (2016). The PREs of the suggested ratio estimators with respect to the prevailing estimators were calculated in Table 3 . It is clearly seen that the suggested estimators outperformed all prevailing estimators in the literature which shows that the proposed estimators are more efficient. Also, the percentage relative efficiency of the proposed estimator revealed more than 100 percent efficiency over the existing ratio estimators. Practical studies revealed that the bias and the mean squared error of the suggested estimators are less than those of the prevailing estimators under the study population. Therefore, the improved ratio estimators suggested in this study should be used for improved results, and should be preferred over the prevailing ratio estimators for empirical applications.

\subsection{Conclusion and Policy Implications}

In this study, we have suggested three improved ratio type estimators for calculating the population mean of the study variable $\mathrm{Y}$ using a linear mixture of the coefficient of variation, kurtosis, and skewness and variance information of the auxiliary variable X. Practical studies revealed that the relative constant, bias and the mean squared error of the suggested estimators were lower than that of the prevailing estimators under the study population. We have also shown that the suggested estimators are more productive than the existing estimators in the literature based on the PRE values. These improved ratio estimators will go a long way in formulating economic, business, banking, demographic and market policies based on reduced errors of the estimates gotten by adopting these estimators. 


\section{References}

Abid, M. Nasir, A. Hafiz, Z. N. and Zhengyan, L. (2016). Enhancing the mean ratio estimators for estimating population mean using non-conventional location parameter. Revista Colombiana DE Estadistica, pp. 63-79.

Adepoju, K. A. and Shittu, O. I. (2013). On the efficiency of ratio estimator based on linear combination of median, coefficients of skewness and kurtosis. American Journal of Mathematics and Statistics, pp. 130-134.

Cochran, W. G. (1940). The estimation of the yields of the cereal experiments by sampling for the ratio of grain to total produce. Journal of Agricultural Science, pp. 262-275.

Cochran, W. G. (1977). Sampling techniques. New York: John Wiley and Sons.

Jeelani, M. I. Maqbool, S. and Mir, S. A. (2013). Modified ratio estimators of population mean using linear combination of coefficient of skewness and quartile deviation. International Journal of Modern Mathematical Sciences, pp. 174-183.

Kadilar, C. and Cingi, H. (2004). Ratio estimators in simple random sampling. Applied Mathematics and Computation, pp. 893-902.

Kadilar, C. and Cingi, H. (2006). An Improvement in estimating the population mean by using the correlation co-efficient. Hacettepe Journal of Mathematics and Statistics, pp. 103-109.

Koyuncu, N. and Kadilar, C. (2009). Efficient estimators for the population mean. Hacettepe journal of mathematics and statistics, pp. 217-225.

Murthy, M. N. (1967). Sampling theory and methods. Calcutta India: Statistical Publishing Society.

Pandey, B. N. and Dubey, V. ( 1988). Modified product estimator using coefficient of variation of auxiliary variate. Assam Statistical Review, pp.64-66.

Prasad, B. (1989). Some improved ratio type estimators of population mean and ratio in finite population sample surveys. Communications in Statistics: Theory and Methods, pp. 379-392.

Sen, A. R. (1993). Some early developments in ratio estimation. Biometric Journal, pp. 3-13.

Shittu, O. I. and Adepoju, K. A. (2014). On the efficiency of modified ratio estimator based on linear combination of kurtosis, median and quartile deviation. International Journal of Modern Mathematical Sciences, pp. 103-107. 
Singh, G. N. (2003). On the improvement of product method of estimation in sample surveys. Journal of the Indian Society of Agricultural Statistics, pp. 267-265.

Singh, H. P. and Tailor, R. (2003). Use of known correlation co-efficient in estimating the finite population means. Statistics in Transition, pp. 555-560.

Singh, H. P. and Tailor, R. (2005). Estimation of finite population mean with known co-efficient of variation of an auxiliary. Statistica, pp. 301-313.

Singh, H. P. Tailor, R. and Kakran, M. S. (2004). An improved estimator of population means using power transformation. Journal of the Indian Society of Agricultural Statistics, pp. 223-230.

Sisodia, B. V. and Dwivedi, V. K. (1981). A modified ratio estimator using coefficient of variation of auxiliary variable. Journal of the Indian Society of Agricultural Statistics, pp. 13-18.

Upadhyaya, L. N. and Singh, H. (1999). Use of transformed auxiliary variable in estimating the finite populations mean. Biometrical Journal, pp. 627-636.

Wolter, K. M. (1985). Introduction to variance estimation. Berlin: SpringerVerlag.

Yan, Z. and Tian, B. (2010). Ratio method to the mean estimation using coefficient of skewness of auxiliary variable. Communications in Computer and Information Science, pp. 103-110. 


\section{APPENDICES}

\section{Notations}

\begin{tabular}{|c|c|}
\hline$N$ & Population size \\
\hline$n$ & Sample size \\
\hline$f=\frac{n}{N}$ & Sampling fraction \\
\hline$Y$ & Study/main variable \\
\hline$X$ & Auxiliary/supporting variable \\
\hline $\bar{X}$ & Mean of $X$ \\
\hline $\bar{Y}$ & Mean of $Y$ \\
\hline $\bar{x}$ & Sample mean of $x$ \\
\hline $\bar{y}$ & Sample mean of $y$ \\
\hline$S_{x}, S_{y}$ & Variances of $X$ and $Y$ \\
\hline$S_{y x}$ & Covariance between variables $X$ and $Y$ \\
\hline$C_{x}, C_{y}$ & Coefficient of variation $X$ and $Y$ \\
\hline$\rho$ & Correlation coefficient of $X$ and $Y$ \\
\hline$\beta()$. & Bias of the estimator \\
\hline$M S E()$. & Mean square error of the estimator \\
\hline$P R E$ & Percentage relative efficiency \\
\hline$\tilde{Y}_{i}$ & abid et al. (2016) existing modified ratio estimator of $\bar{Y}$ \\
\hline $\bar{Y}_{p i s}$ & existing modified ratio estimator of $\bar{Y}$ \\
\hline $\bar{Y}_{p a i}$ & existing modified ratio estimator of $\bar{Y}$ \\
\hline$M d$ & Median of $X$ \\
\hline$\beta_{1}$ & Population skewness \\
\hline$\beta_{2}$ & Population kurtosis \\
\hline$D$ & Deciles \\
\hline$D M$ & Decile mean \\
\hline$Q D$ & Quartile Deviation \\
\hline$T M$ & Tri-mean \\
\hline$H L$ & Hodges-Lehmann estimator (Median) \\
\hline$M R$ & Mid-range \\
\hline$K t$ & Kurtosis \\
\hline$S k$ & Skewness \\
\hline$C v$ & Coefficient of variation \\
\hline
\end{tabular}

Subscript $i, j$ for existing estimators. 